\title{
Linearized Stability of Bardeen de-Sitter Thin-Shell Wormholes
}

\author{
Hassan Alshal ${ }^{1,2, \varpi}$ \\ ${ }^{1}$ Department of Physics, Faculty of Science, Cairo University, Giza, 12613, Egypt. \\ ${ }^{2}$ Department of Physics, University of Miami, Coral Gables, FL 33146, USA.
}

\begin{abstract}
A thin-shell wormhole is crafted by the cut-and-paste method of two Bardeen de-Sitter black holes using Darmois-Israel formalism. Dynamics and stability of the wormhole is also studied around the static solutions of the linearized radial perturbations at the throat of the wormhole.

Keywords: Bardeen; black hole; de-Sitter; thin-shell; wormhole
\end{abstract}

\section{INTRODUCTION}

The first wormhole solution was discovered by Ludwig Flamm [1]. It was rediscovered as the EinsteinRosen bridge [2]. Einstein and Rosen introduced their bridge to develop a non-Boscovichian, i.e. singularity-free, atomic model of gravity and electromagnetism. Later, Wheeler developed a theory about geons [3], a topologi-cally unstable gravitoelectromagnetic quasisoliton that can connect widely separated spacetime regions. Misner and Wheeler tried to develop the theory of geons into a geometrical unified classical theory [4]. In Misner and Wheeler project the wormhole term was coined.

Between the development of geons and the rejuvenation of traversable wormhole studies by Morris and Thorne [5], Ellis studied the flow of "substantial ether" through a drainhole [6]. Also Bronnikov analyzed tunnel-like solutions [7], which are considered precursors to the studies of wormholes in modified theories of gravity [8]. More on wormholes can be found in Ref. [9]. Geons reappear again in galileon theory as a scalartensor theory [10]. Even in Euclidean space, Ellis variant p-norm drainholes can be used in pedagogical examples to study electrostatics $\llbracket 11-14]$.

To find a wormhole solution to field equation, one can choose some equations of state such as phantom energy [15,

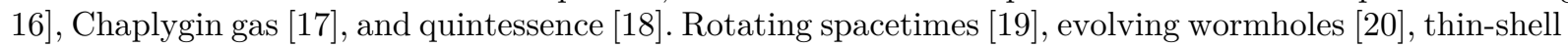
spacetimes [21], and dust shell wormholes [22] are different ways to "ameliorate" the violation of energy conditions associated with the laring-out condition, that is necessary for the field equations to have wormhole solutions. There are numerous studies that consider different black holes in de-Sitter and anti-de-Sitter spacetimes [23-28]. Also thin-shell wormholes can be obtained from regular black holes [29, 30].

In this letter we construct Bardeen de-Sitter thin-shell wormhole. Bardeen black hole [31] is an interesting regular black hole, i.e. with no geometric singularity. Bardeen black hole can be discerned as a quantum-corrected Schwarzschild black hole [32] by applying the generalized uncertainty principle and its corresponding tunneling approach with thermodynamics [33-40]. The Bardeen black hole is imposed in de-Sitter background $(\mathrm{BdS})[41]$. The BdS solution in arbitrary dimensions and the corresponding thermodynamics for each dimension is also considered [42].

In section (II), we use Visser's technique of cut-and-paste [43, 44], together with Darmois-Israel formalism [45], to connect two regions of BdS spacetime through thin shell. We also study the components of the stress-energymomentum surface tensor using the extrinsic curvature. We comment on the violation of energy conditions in terms of stress components. We also calculate the attraction and repulsion nature of the wormhole throat in terms of the acceleration.

In section (III), we analyze the linear stability of BdS thin-shell wormhole by studying the concavity condition on the "speed of sound" as a function in BdS parameters: the mass, the magnetic monopoles and the cosmological constant. And we see the change in stability regions upon varying the amount of magnetic monopoles while both mass and cosmological constant are fixed. In section (IV) we summarize and comment on the results of the previous two sections.

\section{VISSER'S CUT-AND-PASTE TECHNIQUE AND THE DARMOIS-ISRAEL FORMALISM}

The BdS black hole is constructed [41] starting with the metric

$$
d s_{\mathrm{BdS}}^{2}=-\left(1-\frac{2 m(r)}{r}\right) d t^{2}+\left(1-\frac{2 m(r)}{r}\right)^{-1} d r^{2}+r^{2} d \theta^{2}+r^{2} \sin ^{2}(\theta) d \phi^{2}
$$

\footnotetext{
—Electronic address: halshal@sci.cu.edu.eg
} 
The field equations are derived from the action [46]:

$$
\mathcal{A}=\int d^{4} x \sqrt{-g}\left[\frac{R-2 \Lambda}{16 \pi}-\frac{1}{4 \pi} \frac{3 M}{|\mu|^{3}}\left(\frac{\sqrt{2 \mu^{2} F}}{1+\sqrt{2 \mu^{2} F}}\right)^{\frac{5}{2}}\right],
$$

where $\mu$ is the magnetic monopole charge, $\mathrm{M}$ is the mass of the black hole, and $F=\frac{1}{4} F^{\mu \nu} F_{\mu \nu}$. Therefore eq.(1) becomes:

$$
d s_{\mathrm{BdS}}^{2}=-f(r) d t^{2}+f(r)^{-1} d r^{2}+r^{2} d \theta^{2}+r^{2} \sin ^{2}(\theta) d \phi^{2},
$$

where

$$
f(r)=1-\frac{2 M r^{2}}{\left(r^{2}+\mu^{2}\right)^{3 / 2}}-\frac{\Lambda}{3} r^{2} .
$$

And by finding the roots of $f(r)=0$, or the roots of the dedic polynomial

$$
\begin{aligned}
& r^{10} \Lambda^{2}+r^{8}\left(3 \Lambda^{2} \mu^{2}-6 \Lambda\right)+r^{6}\left(3 \Lambda^{2} \mu^{4}-18 \Lambda \mu^{2}+9\right) \\
& +r^{4}\left(\Lambda^{2} \mu^{6}-18 \Lambda \mu^{4}+27 \mu^{2}-36 M^{2}\right)+r^{2}\left(27 q^{4}-6 \Lambda \mu^{6}\right)+9 \mu^{6}=0,
\end{aligned}
$$

one can determine the location of the inner, event $\left(r_{h}\right)$ and cosmological $\left(r_{c}\right)$ horizons of the BdS. However, The combinations of $M, \mu$, and $\Lambda$ that lead to formation extreme BdS - at which the event and cosmological horizons coincide by setting $f(r)=f^{\prime}(r)=0$-must be avoided [26] so the throat radius $a$ of the wormhole exists as $r_{h}<a<r_{c}$.

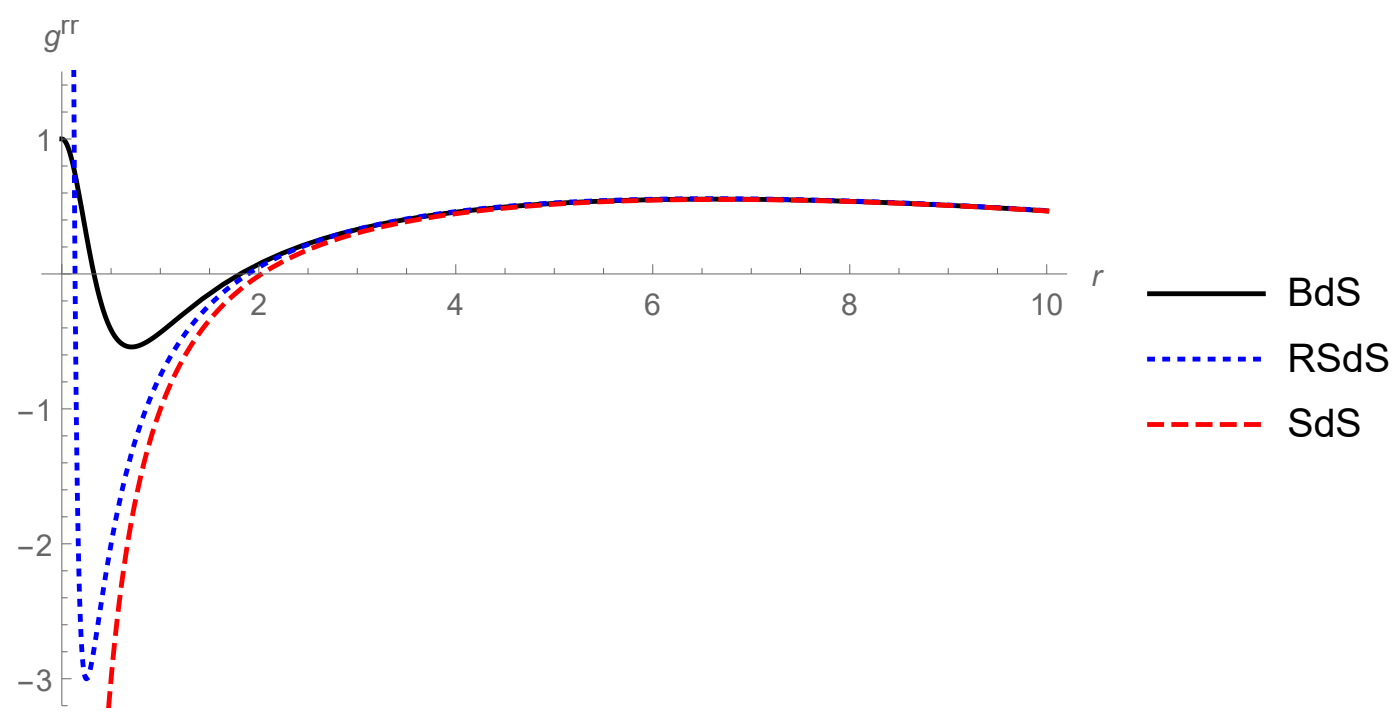

Figure 1: The behavior of $g^{r r}$ metric component for Bardeen de-Sitter (BdS: $M=1, \Lambda=0.01, \mu=0.5$ ), ReissnerNordstrom de-Sitter (RNdS: $M=1, \Lambda=0.01, Q=0.5$ ) and Schwarzschild de-Sitter (SdS: $M=1, \Lambda=0.01$ ).

Following the Cut-and-Paste technique [43, 47], one can easily construct a geodesically complete manifold $\Gamma=\Gamma_{+} \bigcup \Gamma_{-}$by pasting the region of timelike hypersurfaces that is called thin shell $\partial \Gamma=\partial \Gamma_{+} \bigcup \partial \Gamma_{-}$, where $\partial \Gamma_{ \pm}:=\left\{r_{ \pm}=a \mid a>r_{h}\right\}$, that bounds the bulk of two BdS cutting spacetimes $\Gamma_{ \pm}:=\left\{r_{ \pm} \leq a \mid a>r_{h}\right\}$ inside the throat radius $a$.

Now we follow Darmois-Israel formalism [48, 49] by defining the coordinates of $\Gamma$ as $x^{\mu}:=(t, r, \theta, \phi)$ and the coordinates of the shell $\partial \Gamma$ as $\zeta^{i}:=(\tau, \theta, \phi)$, where $\tau$ is the proper time that a comoving frame measures on the throat of the wormhole. The induced metric of the shell is:

$$
d s_{\partial \Gamma}^{2}=-d \tau^{2}+r^{2} d \theta^{2}+r^{2} \sin ^{2}(\theta) d \phi^{2}
$$

where the parametric equation that relates $\Gamma$ to $\partial \Gamma$ is $r=a(\tau)$.

Then Gauss-Kodazzi decomposition of spacetime yields Israel's junction condition on $\Gamma$. The condition is described by the energy momentum tensor on the shell $\mathcal{S}_{j}^{i}=\operatorname{diag}\left(-\sigma, p_{\theta}, p_{\phi}\right)$ as

$$
\mathcal{S}_{j}^{i}=-\frac{1}{8 \pi}\left(\left[\mathcal{K}_{j}^{i}\right]-\delta_{j}^{i} \mathcal{K}\right),
$$


where the $\mathcal{K}_{j}^{i}$ is the extrinsic curvature, $\left[\mathcal{K}_{j}^{i}\right]=\mathcal{K}_{j}^{i+}-\mathcal{K}_{j}^{i-}$, and $\mathcal{K}=\left[\mathcal{K}_{i}^{i}\right]$.

We define the unit vectors $n_{\mu}^{ \pm}$normal to $\partial \Gamma$ as

$$
n_{\mu}^{ \pm}= \pm\left(\left|g^{\alpha \beta} \frac{\partial f}{\partial x^{\alpha}} \frac{\partial f}{\partial x^{\beta}}\right|^{-1 / 2} \frac{\partial f}{\partial x^{\mu}}\right) .
$$

Then The extrinsic curvature, or the second fundamental form, is defined in terms of the unit vectors as

$$
\mathcal{K}_{i j}^{ \pm}=-n_{\mu}\left(\frac{\partial^{2} x^{\mu}}{\partial \zeta^{i} \zeta^{j}}+\Gamma_{\nu \rho}^{\mu \pm} \frac{\partial x^{\nu}}{\partial \zeta^{i}} \frac{\partial x^{\rho}}{\partial \zeta^{j}}\right)
$$

Then we substitute eq.(4) in eq.(8) to get:

$$
n_{\mu}^{ \pm}=\left(\mp \dot{a}, \pm \frac{\sqrt{\dot{a}^{2}+f(a)}}{f(a)}, 0,0\right) .
$$

So, we substitute eq. 10 in eq.99 to get the components of the extrinsic curvature as

$$
\begin{aligned}
& \mathcal{K}_{\theta}^{\theta \pm}=\mathcal{K}_{\phi}^{\phi \pm}= \pm \frac{1}{a} \sqrt{1-\frac{2 M a^{2}}{\left(a^{2}+\mu^{2}\right)^{3 / 2}}-\frac{\Lambda}{3} a^{2}+\dot{a}^{2}}, \\
& \mathcal{K}_{\tau}^{\tau \pm}= \pm \frac{\frac{6 M a^{3}}{\left(\mu^{2}+a^{2}\right)^{5 / 2}}-\frac{4 M a}{\left(\mu^{2}+a^{2}\right)^{3 / 2}}-\frac{2 \Lambda a}{3}+\ddot{a}}{\sqrt{1-\frac{2 M a^{2}}{\left(a^{2}+\mu^{2}\right)^{3 / 2}}-\frac{\Lambda}{3} a^{2}+\dot{a}^{2}}} .
\end{aligned}
$$

Then, we use the the last results to define the surface stresses as

$$
\begin{gathered}
\sigma=-\frac{1}{\pi} \mathcal{K}_{\theta}^{\theta}=-\frac{1}{2 \pi a} \sqrt{1-\frac{2 M a^{2}}{\left(a^{2}+\mu^{2}\right)^{3 / 2}}-\frac{\Lambda}{3} a^{2}+\dot{a}^{2}}, \\
p=p_{\theta}=p_{\phi}=\frac{1}{8 \pi}\left(\mathcal{K}_{\tau}^{\tau}+\mathcal{K}_{\theta}^{\theta}\right)=\frac{3}{8 \pi a} \frac{\frac{1}{3}-\frac{2 M a^{2}}{\left(a^{2}+\mu^{2}\right)^{3 / 2}}-\frac{\Lambda}{3} a^{2}+\frac{2 M a^{4}}{\left(a^{2}+\mu^{2}\right)^{5 / 2}}+a \ddot{a}+\dot{a}^{2}}{\sqrt{1-\frac{2 M a^{2}}{\left(a^{2}+\mu^{2}\right)^{3 / 2}}-\frac{\Lambda}{3} a^{2}+\dot{a}^{2}}} .
\end{gathered}
$$

And for the static configuration, i.e. $\dot{a}=\ddot{a}=0$, the surface stress become

$$
\begin{gathered}
\sigma_{0}=-\frac{1}{2 \pi a_{0}} \sqrt{1-\frac{2 M a_{0}^{2}}{\left(a_{0}^{2}+\mu^{2}\right)^{3 / 2}}-\frac{\Lambda}{3} a_{0}^{2}}, \\
p_{0}=\frac{1}{8 \pi}\left(\mathcal{K}_{\tau}^{\tau}+\mathcal{K}_{\theta}^{\theta}\right)=\frac{3}{8 \pi a_{0}} \frac{1-\frac{2 M a_{0}^{2}}{\left(a_{0}^{2}+\mu^{3}\right)^{3 / 2}}-\frac{\Lambda}{3} a_{0}^{2}+\frac{2 M a_{0}^{4}}{\left(a_{0}^{2}+\mu^{2}\right)^{5 / 2}}}{\sqrt{1-\frac{2 M a_{0}^{2}}{\left(a_{0}^{2}+\mu^{2}\right)^{3 / 2}}-\frac{\Lambda}{3} a_{0}^{2}}}-\frac{1}{4 \pi a_{0}} \frac{1}{\sqrt{1-\frac{2 M a_{0}^{2}}{\left(a_{0}^{2}+\mu^{2}\right)^{3 / 2}}-\frac{\Lambda}{3} a_{0}^{2}}} .
\end{gathered}
$$

From the last two equations, surface density $\sigma_{0}$ leads to the violation of the weak energy condition (WEC). Meanwhile, the null energy condition (NEC), $\sigma_{0}+p_{0}>0$, can be maintained with no need to any exotic matter as long as $f\left(a_{0}\right)<\frac{6 M a_{0}^{4}}{\left(a_{0}^{2}+\mu^{2}\right)^{5 / 2}}$. And for the strong energy condition (SEC), $\sigma_{0}+3 p_{0}>0$, it is also maintained with $f\left(a_{0}\right)<\frac{9 M a^{4}}{\left(\mu^{2}+a^{2}\right)^{5 / 2}}-\frac{6 M a^{2}}{\left(\mu^{2}+a^{2}\right)^{3 / 2}}-\Lambda a^{2}$.

For a BdS black hole with a mass density that it localized at the throat $\rho=\sigma_{0} \delta\left(r-a_{0}\right)$ and no radial pressure $p_{r}=0$, The total amount of exotic matter necessary to keep the wormhole open is

$$
\Omega_{\sigma}=\int_{0}^{2 \pi} \int_{0}^{\pi} \int_{\infty}^{-\infty} \sqrt{-g} \sigma_{0} \delta\left(r-a_{0}\right) d r d \theta d \phi=-2 a_{0} \sqrt{1-\frac{2 M a_{0}^{2}}{\left(a_{0}^{2}+\mu^{2}\right)^{3 / 2}}-\frac{\Lambda}{3} a_{0}^{2}} .
$$

We can examine the attractive and repulsive characters of the constructed thin-shell wormhole by studying the four-acceleration $a^{\mu}=u^{\nu} \nabla_{\nu} u^{\mu}$, where $u^{\mu}=(1 / \sqrt{f(r)}, 0,0,0)$. The geodesic equation of a test particle is

$$
\frac{d^{2} r}{d \tau^{2}}=-a^{r}
$$

where the radial acceleration is given by

$$
a^{r}=\Gamma_{t t}^{r}\left(\frac{d t}{d \tau}\right)^{2}=\frac{M r^{3}}{\left(r^{2}+\mu^{2}\right)^{5 / 2}}-\frac{\Lambda}{3} r-\frac{2 M \mu^{2} r}{\left(r^{2}+\mu^{2}\right)^{5 / 2}} .
$$

We notice that the wormhole has attractive or repulsive nature if $a^{r}>0$ or $a^{r}<0$ respectively. 

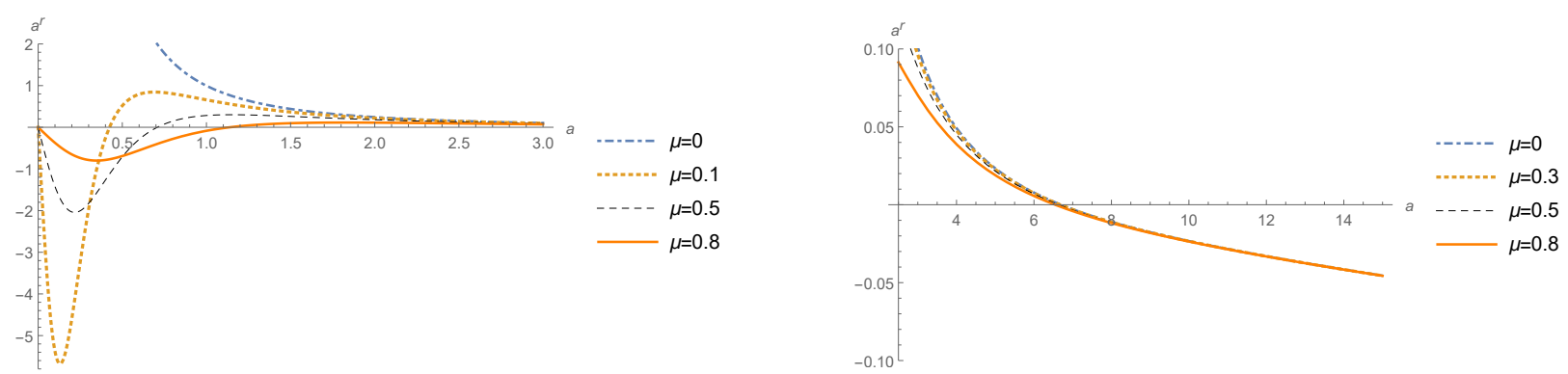

Figure 2: Attraction and repulsion in terms of acceleration $a^{r}$ vs. the throat radius $a$ with fixed $M=1$ and $\Lambda=0.01$, and different values of magnetic monopole $\mu$.

\section{LINEARIZED STABILITY ANALYSIS}

The stability of the wormhole can be checked 47] by performing linear perturbation about the static configuration $a=a_{0}$ for eq.(14) and eq.(15). One can easily notice that differentiating eq.(12) with respect to $\tau$ yields the continuity equation

$$
\frac{d(\sigma A)}{d \tau}+p \frac{d A}{d \tau}=0
$$

which directly leads to

$$
\sigma^{\prime}=-\frac{2}{a}(\sigma+p,)
$$

where $A=4 \pi a^{2}$ is the area of the wormhole throat, $\sigma^{\prime}=\dot{\sigma} / \dot{a}$, the dot means $d / d \tau$, and the prime means $d / d a$. If we rearrange eq. $(12)$, we define a potential function

$$
V(a)=f(a)-4 \pi^{2} a^{2} \sigma^{2}=-\dot{a} .
$$

Then we substitute with eq.20 in the first derivative of eq. (21) to get

$$
V^{\prime}(a)=\frac{6 M a^{3}}{\left(\mu^{2}+a^{2}\right)^{5 / 2}}-\frac{4 M a}{\left(\mu^{2}+a^{2}\right)^{3 / 2}}-\frac{2 \Lambda a}{3}+8 \pi^{2} a \sigma(\sigma+2 p) .
$$

And for the second derivative of (21), we parameterize the pressure to be a function in the density $p:=p(\sigma)$ [21]. Then we introduce a new parameter $\vartheta(\sigma)=d p / d \sigma$, which can be seen as the "speed of sound". And the second derivative of 21 becomes

$$
\begin{aligned}
V^{\prime \prime}(a)= & f^{\prime \prime}(a)-8 \pi^{2}\left[2 \sigma(\sigma+p)(1+2 \vartheta)+(\sigma+2 p)^{2}\right] \\
& =f^{\prime \prime}(a)+\left[\frac{1}{a^{2}}\left(a f^{\prime}(a)-2 f(a)\right)(1+2 \vartheta)-\frac{1}{2}\left(\frac{f^{\prime}(a)}{f(a)}\right)^{2}\right] .
\end{aligned}
$$

To linearize the model, we apply Taylor expansion to the potential function around the static point $a=a_{0}$ such that eq. 21) becomes

$$
V(a)=V\left(a_{0}\right)+\left(a-a_{0}\right) V^{\prime}\left(a_{0}\right)+\frac{1}{2}\left(a-a_{0}\right)^{2} V^{\prime \prime}\left(a_{0}\right)+\mathcal{O}\left[\left(a-a_{0}\right)^{3}\right] .
$$

We use eq.14 and eq.15 to evaluate eq.21 and eq.22 at $a=a_{0}$. Therefore, we get $V\left(a_{0}\right)=V^{\prime}\left(a_{0}\right)=0$. Meanwhile eq. 23 becomes

$$
\begin{aligned}
V^{\prime \prime}\left(a_{0}\right)= & \frac{30 a_{0}^{2} M}{\left(a_{0}^{2}+\mu^{2}\right)^{5 / 2}}-\frac{4 M}{\left(a_{0}^{2}+\mu^{2}\right)^{3 / 2}}-\frac{30 a_{0}^{4} M}{\left(a_{0}^{2}+\mu^{2}\right)^{7 / 2}}-\frac{2 \Lambda}{3} \\
& -\frac{1}{a_{0}^{2}}(1+2 \vartheta)\left(\frac{6 M a_{0}^{2}}{\left(\mu^{2}+a_{0}^{2}\right)^{5 / 2}}-\frac{2}{a_{0}^{2}}\right) \\
& -\frac{1}{2}\left(\frac{-\frac{4 M a_{0}}{\left(\mu^{2}+a_{0}^{2}\right)^{3 / 2}}+\frac{6 M a_{0}^{3}}{\left(\mu^{2}+a_{0}^{2}\right)^{5 / 2}}-\frac{2 \Lambda a_{0}}{3}}{1-\frac{2 M a_{0}^{2}}{\left(\mu^{2}+a_{0}^{2}\right)^{3 / 2}}-\frac{\Lambda a_{0}^{2}}{3}}\right)^{2} .
\end{aligned}
$$


Of course we can use $(1+2 \vartheta)=\left(\sigma^{\prime}+2 p^{\prime}\right) / \sigma^{\prime}$ to express $\vartheta$ in terms of the metric parameters $M, \mu$, and $a$. But we will not as we need to study the behavior of $\vartheta$ when the throat is stable.

The concave down condition $V^{\prime \prime}\left(a_{0}\right)<0$ results in provoking either expansion or contraction of the throat when any small perturbation occurs. While the convex, or the concave up, condition $V\left(a_{0}\right)^{\prime \prime}>0$ stabilizes the throat with a local minimum of $V\left(a_{0}\right)$ at $a_{0}$. Therefore, we solve for $\vartheta_{0}$ at that local minimum to get

$$
\begin{aligned}
\vartheta_{0}<\frac{1}{2}\left\{1-\frac{a_{0}^{2}}{\left(\frac{6 M a_{0}^{2}}{\left(\mu^{2}+a_{0}^{2}\right)^{5 / 2}}-\frac{2}{a_{0}^{2}}\right)}\left[\frac{30 a_{0}^{2} M}{\left(a_{0}^{2}+\mu^{2}\right)^{5 / 2}}-\frac{4 M}{\left(a_{0}^{2}+\mu^{2}\right)^{3 / 2}}-\frac{30 a_{0}^{4} M}{\left(a_{0}^{2}+\mu^{2}\right)^{7 / 2}}-\frac{2 \Lambda}{3}\right.\right. \\
\\
\left.\left.-\frac{1}{2}\left(\frac{-\frac{4 M a_{0}}{\left(\mu^{2}+a_{0}^{2}\right)^{3 / 2}}+\frac{6 M a_{0}^{3}}{\left(\mu^{2}+a_{0}^{2}\right)^{5 / 2}}-\frac{2 \Lambda a_{0}}{3}}{1-\frac{2 M a_{0}^{2}}{\left(\mu^{2}+a_{0}^{2}\right)^{3 / 2}}-\frac{\Lambda a_{0}^{2}}{3}}\right)\right]\right\}
\end{aligned}
$$

Or

$$
\vartheta_{0}<\frac{1}{2}\left(1-\frac{-\frac{4 \Lambda a_{0}^{2}\left(6 \mu^{2} M-3 M a_{0}^{2}+\Lambda\left(\mu^{2}+a_{0}^{2}\right)^{5 / 2}\right)^{2}}{3\left(\mu^{2}+a_{0}^{2}\right)^{2}\left(6 M a_{0}^{2}+\left(\Lambda a_{0}^{2}-3\right)\left(\mu^{2}+a_{0}^{2}\right)^{3 / 2}\right)^{2}}+\frac{30 M a_{0}^{2}}{\left(\mu^{2}+a_{0}^{2}\right)^{5 / 2}}-\frac{4 M}{\left(\mu^{2}+a_{0}^{2}\right)^{3 / 2}}-\frac{30 M a_{0}^{4}}{\left(\mu^{2}+a_{0}^{2}\right)^{7 / 2}}}{\frac{6 M}{\left(\mu^{2}+a_{0}^{2}\right)^{5 / 2}}-\frac{2}{a_{0}^{4}}}\right) .
$$
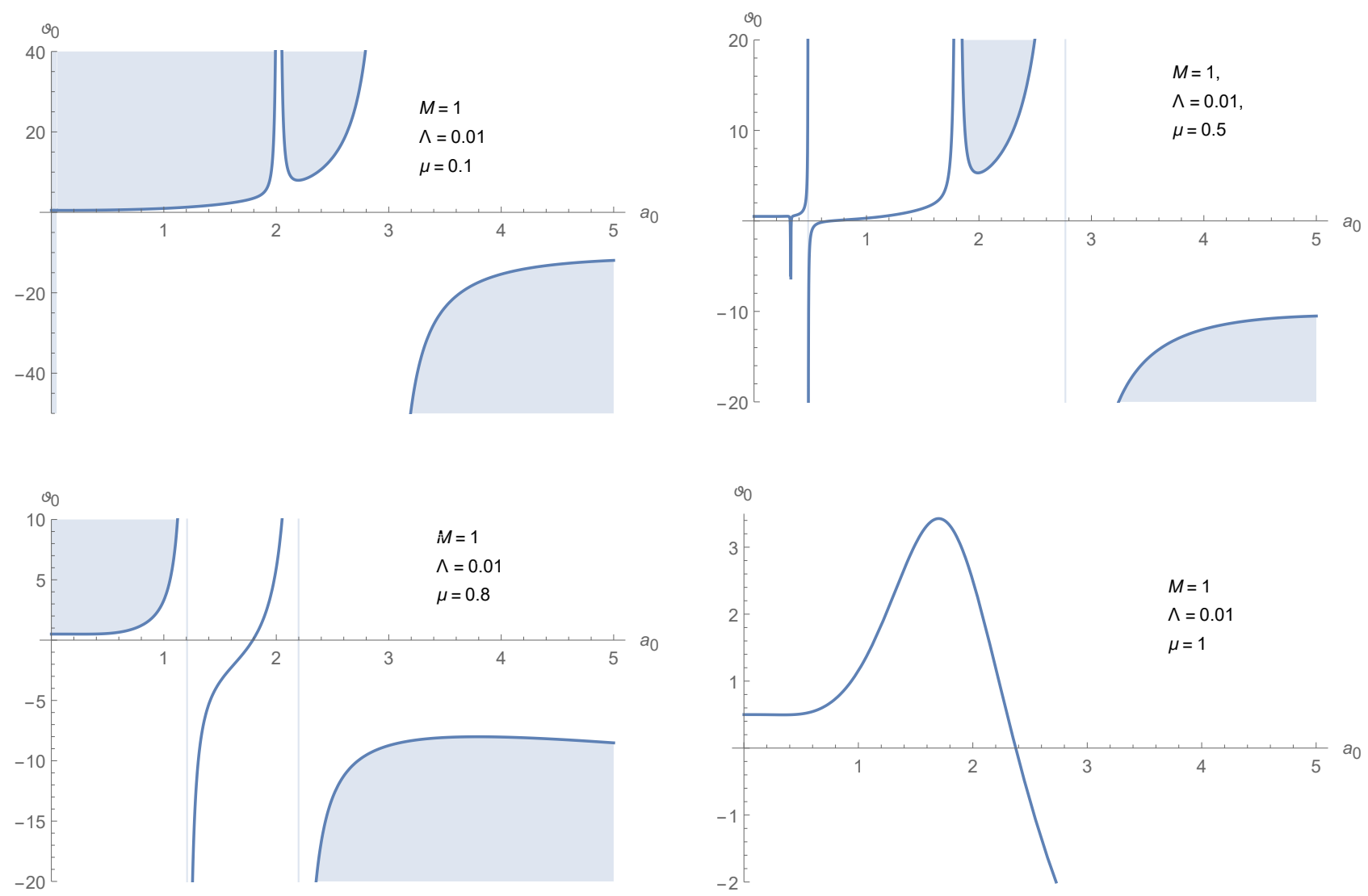

Figure 3: Regions of stability of the thin-shell wormhole for the Bardeen de-Sitter solution for fixed values of $M$ and $\Lambda$, and different values of $\mu$. Stable regions are the blue shaded domains.

\section{DISCUSSION}

In this letter we construct Bardeen de-Sitter thin-shell wormhole. We use Visser's technique of cut-and-paste with Darmois-Israel formalism to connect two regions of BdS spacetime through a thin shell. We compare the asymptotic behavior of the metric with that of SdS and RNdS as in fig.11. We also study the components of the stress-energy-momentum surface tensor using the extrinsic curvature. We find that WEC is always violated. However, both NEC and SEC can be maintained upon considering conditions on the $f(r)$. We also calculate the attractive and repulsive nature of the wormhole throat in terms of the acceleration. 
We analyze the linear stability of BdS thin-shell wormhole by studying the concavity condition on the "speed of sound" as a function in BdS parameters: the mass, the magnetic monopoles and the cosmological constant. And we see the change in stability regions upon varying the charge of magnetic monopoles while both mass and cosmological constant are fixed. The analysis is demonstrated in fig.(3). We conclude that for minute cosmological constant and small amount of magnetic charge, relative to the amount of mass, we find different regions of stability. Once the mass is equal to the magnetic charge, we no longer have stability regions. So we choose different values for magnetic charge that are less than the mass. The results are plotted in fig.(2).

[1] L. Flamm. Physikalische Zeitschrift,17 , 448 (1916). Replicated: Gen. Relativ. Gravit., (2015) 47:72.

[2] A. Einstein and N. Rosen, Phys. Rev. 48, 73 (1935).

[3] J. A. Wheeler, Phys. Rev. 97, 511 (1955).

[4] C. W. Misner and J. A. Wheeler, Annals Phys. 2, 525 (1957).

[5] M. S. Morris and K. S. Thorne, Am. J. Phys. 56, 395 (1988).

[6] H. G. Ellis, J. Math. Phys. 14, 104 (1973).

[7] K. A. Bronnikov, Acta Phys. Polon. B 4, 251 (1973).

[8] F. S. N. Lobo, Fundam. Theor. Phys. 189, pp. (2017).

[9] M. Visser, Woodbury, USA: AIP (1995) 412 p.

[10] T. L. Curtright and D. B. Fairlie, Phys. Lett. B 716, 356 (2012) [arXiv:1206.3616 [hep-th]].

[11] T. Curtright, H. Alshal, P. Baral, S. Huang, J. Liu, K. Tamang, X. Zhang and Y. Zhang, Eur. J. Phys. 40, no. 1, 015206 (2019) [arXiv:1805.11147 [physics.class-ph]].

[12] H. Alshal and T. Curtright, arXiv:1806.03762 [physics.class-ph].

[13] H. Alshal, T. Curtright and S. Subedi, arXiv:1808.08300 [physics.class-ph].

[14] H. Alshal, arXiv:1905.00403 [physics.class-ph].

[15] S. V. Sushkov, Phys. Rev. D 71, 043520 (2005) [gr-qc/0502084].

[16] F. S. N. Lobo, Phys. Rev. D 71, 124022 (2005) [gr-qc/0506001].

[17] F. S. N. Lobo, Phys. Rev. D 73, 064028 (2006) [gr-qc/0511003].

[18] F. S. N. Lobo, Phys. Rev. D 75, 024023 (2007) [gr-qc/0610118].

[19] E. Teo, Phys. Rev. D 58, 024014 (1998) [gr-qc/9803098].

[20] S. Kar and D. Sahdev, Phys. Rev. D 53, 722 (1996) [gr-qc/9506094].

[21] E. Poisson and M. Visser, Phys. Rev. D 52, 7318 (1995) [gr-qc/9506083].

[22] F. S. N. Lobo, Gen. Rel. Grav. 37, 2023 (2005) [gr-qc/0410087].

[23] P. K. F. Kuhfittig, Acta Phys. Polon. B 41, 2017 (2010) [arXiv:1008.3111 [gr-qc]].

[24] F. Rahaman, A. Banerjee and I. Radinschi, Int. J. Theor. Phys. 51, 1680 (2012) [arXiv:1109.0976 [gr-qc]].

[25] M. Sharif and M. Azam, JCAP 1304, 023 (2013) [arXiv:1305.4441 [gr-qc]].

[26] M. Sharif and S. Mumtaz, Adv. High Energy Phys. 2014, 639759 (2014).

[27] A. Eid, New Astron. 39, 72 (2015).

[28] A. Övgün, A. Banerjee and K. Jusufi, Eur. Phys. J. C 77, no. 8, 566 (2017) [arXiv:1704.00603 [gr-qc]].

[29] M. Halilsoy, A. Ovgun and S. H. Mazharimousavi, Eur. Phys. J. C 74, 2796 (2014) [arXiv:1312.6665 [gr-qc]].

[30] M. Sharif and S. Mumtaz, Eur. Phys. J. Plus 132, no. 1, 26 (2017) [arXiv:1604.01012 [gr-qc]].

[31] J. M. Bardeen. in Proc. Int. Conf. GR5, Tbilisi, p. 174 (1968).

[32] R. V. Maluf and J. C. S. Neves, Int. J. Mod. Phys. D 28, no. 03, 1950048 (2018) [arXiv:1801.08872 [gr-qc]].

[33] D. Amati, M. Ciafaloni and G. Veneziano, Phys. Lett. B 216, 41 (1989).

[34] L. J. Garay, Int. J. Mod. Phys. A 10, 145 (1995) [gr-qc/9403008].

[35] F. Scardigli, Phys. Lett. B 452, 39 (1999) [hep-th/9904025].

[36] R. J. Adler, P. Chen and D. I. Santiago, Gen. Rel. Grav. 33, 2101 (2001) [gr-qc/0106080].

[37] A. F. Ali, S. Das and E. C. Vagenas, Phys. Lett. B 678, 497 (2009) [arXiv:0906.5396 [hep-th]].

[38] E. C. Vagenas, A. Farag Ali and H. Alshal, Eur. Phys. J. C 79, no. 3, 276 (2019) [arXiv:1811.06614 [gr-qc]].

[39] E. C. Vagenas, A. F. Ali, M. Hemeda and H. Alshal, Eur. Phys. J. C 79, no. 5, 398 (2019) [arXiv:1903.08494 [hep-th]].

[40] E. C. Vagenas, A. F. Ali and H. Alshal, Phys. Rev. D 99, no. 8, 084013 (2019) [arXiv:1903.09634 [hep-th]].

[41] S. Fernando, Int. J. Mod. Phys. D 26, no. 07, 1750071 (2017) [arXiv:1611.05337 [gr-qc]].

[42] M. S. Ali and S. G. Ghosh, Phys. Rev. D 98, no. 8, 084025 (2018).

[43] M. Visser, Nucl. Phys. B 328, 203 (1989) [arXiv:0809.0927 [gr-qc]].

[44] M. Visser, Phys. Rev. D 39, 3182 (1989) [arXiv:0809.0907 [gr-qc]].

[45] R. Mansouri and M. Khorrami, J. Math. Phys. 37, 5672 (1996) [gr-qc/9608029].

[46] E. Ayon-Beato and A. Garcia, Phys. Lett. B 493, 149 (2000) [gr-qc/0009077].

[47] F. S. N. Lobo and P. Crawford, Class. Quant. Grav. 21, 391 (2004) [gr-qc/0311002].

[48] W. Israel, Nuovo Cim. B 44S10, 1 (1966) [Nuovo Cim. B 44, 1 (1966)] Erratum: [Nuovo Cim. B 48, 463 (1967)].

[49] G. Darmois, Memorial de Sciences Mathematiques, Fascicule XXV, "Les equations de la gravitation einsteinienne", Chapitre V (1927). 